\title{
Has ART Finally Got a Patient-Friendly Progesterone?
}

\author{
Gautam N. Allahbadia ${ }^{1}$
}

Published online: 1 July 2015

(C) Federation of Obstetric \& Gynecological Societies of India 2015

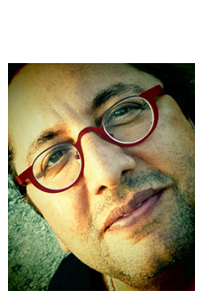

\begin{abstract}
About the Author
Gautam N. Allahbadia, MD (Doctor of Medicine), is the Editor-in-chief of the Journal of Obstetrics and Gynecology of India as well as the IVF Lite (Journal of Minimal Stimulation IVF). He is also the Medical Director of Rotunda-The Center for Human Reproduction, the world-renowned fertility clinic at Bandra, Mumbai, India, as well as the New Hope IVF Clinic at Sharjah, UAE. He is a noted world authority on Ultrasound-guided Embryo Transfers and one of the pioneers in Third Party Reproduction in South-East Asia. Dr. Allahbadia was responsible for India's first trans-ethnic surrogate pregnancy involving a Chinese couple's baby delivered by an unrelated Indian surrogate mother. He has over 100 peer-reviewed publications to his credit and is on the Editorial Board of several international journals. Throughout his career, Dr. Allahbadia has been instrumental in developing new fertility-enhancing protocols and propagating the use of Ultrasound in
\end{abstract} Embryo Transfer procedures. You can read more about his work at www.gautamallahbadia.com.

\section{Introduction}

Progesterone prepares the endometrium for pregnancy by stimulating proliferation in response to human chorionic gonadotropin (hCG), which is produced by the corpus luteum. This occurs in the luteal phase of the menstrual cycle. In assisted reproduction techniques (ART), the progesterone or hCG levels, or both, are low, and the

Gautam N Allahbadia is the Editor-in-chief of the Journal of Obstetrics \& Gynecology of India as well as the IVF Lite (Journal of Minimal Stimulation IVF) and Medical Director at Rotunda-The Center For Human Reproduction, Mumbai, India \& New Hope IVF, Sharjah, UAE.

Gautam N. Allahbadia

ivfwaladoc@gmail.com

1 Rotunda-The Center for Human Reproduction, Mumbai, India natural process is insufficient, so the luteal phase is supported with either progesterone, hCG, or gonadotropin-releasing hormone $(\mathrm{GnRH})$ agonists. Luteal phase support improves implantation rate and thus pregnancy rates, but the ideal method is still unclear. A large recent Cochrane review showed a significant effect in favor of progesterone for luteal phase support, favoring synthetic progesterone over micronized progesterone [1]. Overall, the addition of other substances such as estrogen or hCG did not seem to improve outcomes. Those authors also found no evidence favoring a specific route or duration of administration of progesterone. They found that hCG, or hCG plus progesterone, was associated with a higher risk of ovarian hyperstimulation syndrome (OHSS). The use of hCG should therefore be avoided [1]. There were significant results showing beneficial effects from the addition of $\mathrm{GnRH}$ agonist to progesterone in terms of the outcomes of live birth, clinical pregnancy, and ongoing pregnancy. For now, progesterone seems to be the best option as luteal 
phase support, with better pregnancy results when synthetic progesterone is used [1].

\section{Discussion}

Progesterone is a natural steroid hormone controlling women's reproductive functions [2]. In Donor Egg IVF recipients, exogenous progesterone is used for reproducing the endometrial luteal phase-transformative changes [3]. Available progesterone preparations include oral-, vaginal-, oil-based intramuscular (IM) formulations and now aqueous subcutaneous (SC) Progesterone [4-8]. Oral preparations, however, are of limited use in fertility management because of poor bioavailability [7]. Vaginal progesterone achieves adequate endometrial transformation, despite lower circulating levels of progesterone, but is associated with side effects, e.g., vaginal discharge and/or local irritation $[9,10]$. The parenteral intramuscular route reliably achieves serum levels of progesterone encountered in the natural luteal phase but can cause severe discomfort and pain at the injection site [11]. A new aqueous progesterone preparation for subcutaneous (SC) administration, aimed at providing the advantages of existing parenteral preparations without their local tolerability issues, has recently been developed $[12,13]$. Considering the advantages given by the possibility of self-medication, the SC aqueous formulation could offer a convenient alternative for patients on ART treatments.

When GnRH analogs are used for pituitary down regulation, LH secretion is inhibited, and its effect will be continued in the luteal phase [14]. Abysmally low levels of luteal phase LH may be insufficient to promote endometrial maturation to support an early pregnancy. Endogenous LH secretion can be suppressed for as long as 10 days after GnRH agonist treatment $[15,16]$. In order to compensate the low levels, progesterone gel, intramuscular progesterone, and suppository forms of this steroid are used by clinicians. Numerous studies have shown that supplementary hCG increases the risk of OHSS, and therefore, progesterone seems to be the preferred drug [17, 18]. Intramuscular oil-based injections are obviously painful and have side effects like abscess formation. On the other hand, progesterone suppository may cause lower serum levels which may result in poor implantation; however, numerous clinical trials in this field have had paradoxical results [19-21]. Research to find a patient-compliant and patient-preferred progesterone protocol in IVF has been ongoing for the last five decades. In a prospective study of 206 IVF cases in Texas in 1999, intramuscular progesterone (50 mg daily) and Crinone gel $8 \%$ were compared [22]. Vaginal bleeding (11-12 days after ovum pick up) was more frequent in the Crinone gel group; however, serum progesterone in the intramuscular group was higher. Another randomized study from Italy comparing intramuscular and vaginal progesterone showed that progesterone level by using vaginal gel was more stable and gel was comfortably used by the patients [23]. In 2013, a novel aqueous progesterone formulation was developed with three studies published as part of one research paper [24]. The first study was a three-way cross-over, open-label study in 24 postmenopausal women. Comparison of the pharmacokinetic profiles of a single 100-mg dose of aqueous test product administered by subcutaneous (s.c.) and intramuscular (i.m.) injection and an i.m. reference oily product. The second study was a three-way cross-over open label study of 25,50 , and $100 \mathrm{mg}$ s.c. single doses of the aqueous formulation in 12 post-menopausal women. The third study was a Parallel group, observer-blinded study in 25 fertile women administered multiple s.c. 25 and $50 \mathrm{mg}$ doses of the aqueous formulation once daily for 11 days. Baseline-corrected pharmacokinetic parameters were evaluated. Aqueous formulation (100 mg) was promptly absorbed, achieving progesterone peak serum levels at an earlier time than the reference ( $1 \mathrm{~h}$ vs $7 \mathrm{~h} ; p<0.0001)$. Test and reference were bioequivalent in the extent of exposure: confidence intervals for AUC0-t geometric means ratios were within the prespecified 80-125\% limits. Pharmacokinetics was linear over the range of doses studied. Steady state was reached within 4 days of multiple dose treatment. Sator et al. concluded that the novel progesterone aqueous formulation showed similar bioavailability as the reference oil-based product [24]. In these preliminary studies, it was demonstrated that the serum levels of $\mathrm{P}$ achieved with $25 \mathrm{mg}$ were above the threshold necessary for pre-decidualization to occur [25, 26]. In addition, an earlier phase II study [27] performed in 24 healthy subjects provided evidence that this aqueous progesterone administered SC at a daily dose of 25 or $50 \mathrm{mg}$ was effective at priming the endometrial changes seen in the menstrual cycle in the absence of endogenous $\mathrm{P}$. Because of no difference in the endometrial biopsies having been shown between the two doses tested, the lowest dose $(25 \mathrm{mg} /$ days, which corresponds to the physiologic amount produced by the ovary in the midluteal phase [28] was selected for the phase III trials of LPS in assisted reproduction technologies (ART).

Given the reluctance of some patients to use vaginal preparations owing to the messy administration, vaginal discharge, and, rarely, intolerability, as well as the inconvenience and discomfort associated with prolonged IM administration of $\mathrm{P}$ in oil (castor or sesame oil), a watersoluble injectable $\mathrm{P}$ has been developed that may be administered by subcutaneous (SC) injection [12, 13, 24]. This injection is a complex of $\mathrm{P}$ and hydroxypropylb-cyclodextrin in water [13] which has been demonstrated 
to produce adequate endometrial decidualization at a daily dose of 25 or $50 \mathrm{mg}$ in a dose-finding study [27].

Lockwood et al. compared the safety, efficacy, and tolerability of subcutaneous progesterone (Prolutex, $25 \mathrm{mg}$; IBSA Institut Biochimique SA) with vaginal progesterone gel (Crinone, $8 \%$; Merck Serono) for patients subjected to luteal phase support (LPS) in assisted reproduction technologies (ART) [8]. A total of 683 ART patients were randomized into two groups: Prolutex, $25 \mathrm{mg}$ subcutaneously daily $(n=339)$; and Crinone, $90 \mathrm{mg} 8 \%$ gel daily ( $n=344)$. On the day of oocyte retrieval, Prolutex or Crinone gel was begun for LPS and continued for up to 10 weeks. The nonsignificant difference between the groups was $-3.09 \%$ (95\% confidence interval [CI] -9.91 to 3.73), indicating noninferiority of Prolutex to Crinone. Delivery, and live birth rates resulted to be equivalent between the two treatments (26.8 vs. $29.9 \%$ in the Prolutex and Crinone groups, respectively [ITT]; difference -3.10 [95\% CI -9.87 to 3.68$]$ ). No statistically significant differences were reported for any of the other secondary efficacy endpoints, including comfort of usage and overall satisfaction. The study summarized that implantation rate, pregnancy rate, live birth rate, and early miscarriage rate for Prolutex were similar to those for Crinone. The adverse event profiles were similar, and Prolutex was safe and well tolerated [8].

A prospective, open-label, randomized, controlled, parallel-group, multicenter, two-arm, non-inferiority study was performed at eight fertility clinics [29]. A total of 800 women, aged 18-42 years, with a BMI of $\leq 30 \mathrm{~kg} / \mathrm{m}$ [2], with $<3$ prior completed assisted reproductive technology (ART) cycles, exhibiting baseline (Days 2-3) FSH of $\leq 15 \mathrm{IU} / \mathrm{L}$, and undergoing IVF at eight centers (seven private, one academic) in the USA, were enrolled from January 2009 through June 2011. In total, 800 women undergoing IVF were randomized after retrieval of at least three oocytes to an aqueous preparation of progesterone administered subcutaneously (25 mg daily) or vaginal progesterone (100 $\mathrm{mg}$ bid daily). If a viable pregnancy occurred, progesterone treatment was continued up to 12 weeks of gestation. Using a PP analysis, which included all patients who received an embryo transfer (Prolutex $=392$; Endometrin $=390$ ), the ongoing pregnancy rate per retrieval for subcutaneous versus vaginal progesterone was 41.6 versus $44.4 \%$, with a difference between groups of $-2.8 \%(95 \% \mathrm{CI}-9.7,4.2)$, consistent with the non-inferiority of subcutaneous progesterone for luteal phase support. In addition, rates of initial positive $\beta-\mathrm{hCG}$ (56.4\% subcutaneous vs. $59.0 \%$ vaginal; $95 \%$ CI -9.5 , 4.3), clinical intrauterine pregnancy with fetal cardiac activity (42.6 vs. $46.4 \%$; $95 \%$ CI -10.8, 3.2), implantation defined as number of gestational sacs divided by number of embryos transferred (33.2 vs. $35.1 \%$; $95 \% \mathrm{CI}$ $-7.6,4.0)$, live birth (41.1 vs. $43.1 \%$; $95 \%$ CI -8.9, 4.9), and take-home baby (41.1 vs. $42.6 \% ; 95 \% \mathrm{CI}-8.4,5.4)$ were comparable. Both formulations were well tolerated, with no difference in serious adverse events. Analysis with the intention-to-treat population also demonstrated no difference for any outcomes between the treatment groups. Baker et al. concluded that subcutaneous progesterone represents a novel option for luteal phase support in women undergoing IVF who for personal reasons prefer not to use a vaginal preparation or who wish to avoid the side effects of vaginal or IM routes of administration [29].

\section{Conclusion}

Levine [30] and later Yanushpolsky et al. [31] confirmed that patients prefer vaginal over intramuscular progesterone, and this was clearly related to the pain and inconvenience associated with IM injections, which are difficult to self-administer and are painful, even when the injection is administered by a nurse. Contemporary IVF, however, relies otherwise almost entirely on subcutaneous injections for agonist, antagonist, and gonadotropin therapies, and women feel comfortable in self-administering these injections. Also, some women because of cultural and religious beliefs, particularly once a pregnancy has been confirmed, are uneasy and reluctant to insert medication vaginally and are very concerned about the leakage associated with gels and pessaries, fearing that they might have not absorbed completely, and insertion of a vaginal preparation in case of spotting or vaginal bleeding can be unpleasant. In addition, the vaginal manipulation when performed in a not properly clean environment may increase the risk of genital tract infections, which have been shown to be one of the causes of spontaneous abortion [32], preterm births, and poor pregnancy outcome [33] if not treated immediately. This new product may therefore be a patientfriendly alternative for these patients. The option of administering progesterone SC for LPS in ART will broaden the spectrum of available treatments, an advantage for women needing sustained LPS [34] or disliking vaginal treatments for cultural, personal, or medical reasons.

Conflict of interest None.

\section{References}

1. van der Linden $\mathrm{M}$, Buckingham $\mathrm{K}$, Farquhar $\mathrm{C}$, et al. Luteal phase support for assisted reproduction cycles. Cochrane Database Syst Rev. 20115;(10):CD009154. doi: 10.1002/14651858. CD009154.pub2.

2. Warren MP, Shantha S. Uses of progesterone in clinical practice. Int J Fertil Womens Med. 1999;44:96-103.

3. Gibbons WE, Toner JP, Hamacher P, et al. Experience with a novel vaginal progesterone preparation in a donor oocyte program. Fertil Steril. 1998;69:96-101. 
4. Stanczyk FZ. Pharmacokinetics of progesterone administered by the oral and parenteral routes. J Reprod Med. 1999;44:141-7.

5. Nahoul K, Dehennin L, Jondet M, et al. Profiles of plasma estrogens, progesterone and their metabolites after oral or vaginal administration of estradiol or progesterone. Maturitas. 1993;16: 185-202.

6. Miles RA, Paulson RJ, Lobo RA, et al. Pharmacokinetics and endometrial tissue levels of progesterone after administration by intramuscular and vaginal routes: a comparative study. Fertil Steril. 1994;62:485-90.

7. Simon JA, Robinson DE, Andrews MC, et al. The absorption of oral micronized progesterone: the effect of food, dose proportionality, and comparison with intramuscular progesterone. Fertil Steril. 1993;60:26-33.

8. Lockwood G, Griesinger G, Cometti B, et al. Subcutaneous progesterone versus vaginal progesterone gel for luteal phase support in in vitro fertilization: a noninferiority randomized controlled study. Fertil Steril. 2014;101(1):112.e3-119.e3. doi: 10.1016/j.fertnstert.2013.09.010 (Epub 2013 Oct 17).

9. Blake EJ, Norris PM, Dorfman SF, et al. Single and multidose pharmacokinetic study of a vaginal micronized progesterone insert (Endometrin) compared with vaginal gel in healthy reproductive aged female subjects. Fertil Steril. 2010;94:1296301.

10. Levy T, Yairi Y, Bar-Hava I, et al. Pharmacokinetics of the progesterone-containing vaginal tablet and its use in assisted reproduction. Steroids. 2000;65:645-9.

11. Nillius SJ, Johansson ED. Plasma levels of progesterone after vaginal, rectal, or intramuscular administration of progesterone. Am J Obstet Gynecol. 1971;110:470-7.

12. Zoppetti G, Puppini N, Ospitali F, et al. Pharmaceutics, preformulation and drug delivery. J Pharmaceut Sci. 2007;97:1729-36.

13. Zoppetti G, Puppini N, Pizzuti M, et al. Water soluble progesterone-hydroxypropyl- $\beta$-cyclodextrin complex for injectable formulations. J Incl Phenom Macrocycl Chem. 2007;57:283-8.

14. Speroff L, Fritz MA. Clinical gynecologic endocrinology and infertility. 7th ed. Philadelphia: Wilkins, Lippincott and Williams; 2005.

15. Pritts EA, Atwood AK. Luteal phase support in infertility treatment: a meta-analysis of the randomized trials. Hum Reprod. 2002;17:2287-99.

16. Beckers NG, Macklon NS, Eijkemans MJ, et al. Non-supplemented luteal phase characteristics after the administration of recombinant human chorionic gonadotropin, recombinant luteinizing hormone, or gonadotropin releasing hormone $(\mathrm{GnRH})$ agonist to induce final oocyte maturation in in vitro fertilization patients after ovarian stimulation with recombinant follicle stimulating hormone and $\mathrm{GnRH}$ antagonist co-treatment. J Clin Endocrinol Metab. 2003;88:4186-92.

17. Buvat J, Marcolin G, Guittard C, et al. Luteal support after luteinizing hormone-releasing hormone agonist for in vitro fertilization: superiority of human chorionic gonadotropin over oral progesterone. Fertil Steril. 1990;53:490-4.

18. Mochtar MH, Hogerzeil HV, Mol BW. Progesterone alone versus progesterone combined with HCG as luteal support in GnRHa/ HMG induced IVF cycles: a randomized clinical trial. Hum Reprod. 1996;11:1602-5.

19. Tavaniotou A, Smitz J, Bourgain C, et al. Comparison between different routes of progesterone administration as luteal phase support in infertility treatments. Hum Reprod Update. 2000;6: 139-48.

20. Bourgain C, Smitz J, Camus M, et al. Human endometrial maturation is markedly improved after luteal supplementation of gonadotrophin-releasing hormone analogue/human menopausal gonadotrophin stimulated cycles. Hum Reprod. 1994;9:32-40.

21. Ludwig M, Diedrich K. Evaluation of an optimal luteal phase support protocol in IVF. Acta Obstet Gynecol Scand. 2001;80: 452-66.

22. Chantilis SJ, Zeitoun KM, Patel SI, et al. Use of crinone vaginal progesterone gel for luteal support in in vitro fertilization cycles. Fertil Steril. 1999;72:823-9.

23. Artini PG, Volpe A, Angioni S, et al. A comparative, randomized study of three different progesterone support of the luteal phase following IVF/ET program. J Endocrinol Invest. 1995;18:51-6.

24. Sator M, Radicioni M, Cometti B, et al. Pharmacokinetics and safety profile of a novel progesterone aqueous formulation administered by the s.c. route. Gynecol Endocrinol. 2013;29(3): $205-8$.

25. Usadi RS, Groll JM, Lessey BA, et al. Endometrial development and function in experimentally induced luteal phase deficiency. J Clin Endocrinol Metab. 2008;93:4058-64.

26. Paulson RJ. Hormonal induction of endometrial receptivity. Fertil Steril. 2011;96:530-5.

27. de Ziegler D, Sator M, Binelli D, et al. A randomized trial comparing the endometrial effects of daily subcutaneous administration of 25 and $50 \mathrm{mg}$ progesterone in aqueous preparation. Fertil Steril. 2013;100:860-6.

28. Strauss JFI. The synthesis and metabolism of steroids hormones. In: Strauss JF, Barbieri RL, editors. Yen and Jaffe's reproductive endocrinology. 5th ed. Philadelphia: Saunders; 2004. p. 125-54.

29. Baker VL, Jones CA, Doody K, et al. A randomized, controlled trial comparing the efficacy and safety of aqueous subcutaneous progesterone with vaginal progesterone for luteal phase support of in vitro fertilization. Hum Reprod. 2014;29(10):2212-20. doi: 10.1093/humrep/deu194 (Epub 2014 Aug 6).

30. Levine H. Luteal support in IVF using the novel vaginal progesterone gel Crinone $8 \%$ : results of an open-label trial in 1184 women from 16 US centers. Fertil Steril. 2000;74:836-7.

31. Yanushpolsky E, Hurwitz S, Greenberg L, et al. HornsteinM. Crinone vaginal gel is equally effective and better tolerated than intramuscular progesterone for luteal phase support in in vitro fertilization-embryo transfer cycles: a prospective randomized study. Fertil Steril. 2010;94:2596-9.

32. Donders GG, Van Bulck B, Caudron J, et al. Relationship of bacterial vaginosis and mycoplasmas to the risk of spontaneous abortion. Am J Obstet Gynecol. 2000;183:431-7.

33. Flynn CA, Helwig AL, Meurer LN. Bacterial vaginosis in pregnancy Lockwood G, Griesinger G, Cometti B; 13 European Centers. Subcutaneous progesterone versus vaginal progesterone gel for luteal phase support in in vitro fertilization: a noninferiority randomized controlled study. Fertil Steril. 2014;101(1):112.e3-119.e3. doi: 10.1016/j.fertnstert.2013.09.010. Epub 2013 Oct 17

34. Engmann L, Benadiva C. Agonist trigger: what is the best approach? Agonist trigger with aggressive luteal support. Fertil Steril. 2012;97:531-3. 